\title{
Frequência semanal de treino como um fator associado a dependência ao exercício em atletas recreacionais
}

\section{La frecuencia de entrenamiento semanal como factor asociado a la dependencia del ejercicio en atletas recreativos}

\section{Weekly training frequency as a predictor of exercise dependence in recreational athletes}

\author{
Freire, G.L.M ${ }^{1,2}$; Fortes, L.S ${ }^{3}$, Moreira, J.A.G.L.F; Alves, J.F.N¹, Souza, M.F.d ${ }^{1}$; Nascimento Junior, \\ J.R.A ${ }^{1}$. \\ ${ }^{1}$ University Federal do Vale do São Francisco, Brazil;; ${ }^{2}$ University State of Maringá, Brazil; 3 \\ University Federal da Paraíba, Brazil.
}

\section{RESUMO}

Este estudo transversal teve como objetivo investigar a associação da idade, frequência de treino e tempo de prática com o grau de dependência ao exercício (DE) de atletas recreacionais. Participaram 159 atletas recreacionais de corrida, musculação e crossfit, com média de idade de 31,59 \pm 7,98 anos, do estado de Pernambuco. Os instrumentos utilizados foram um questionário sociodemográfico para caracterizar a amostra e a Exercise Dependence ScaleRevised para medir a DE. A análise dos dados foi conduzida por meio da Correlação de Spearman e a Regressão Múltipla $(p<0,05)$. Os resultados evidenciaram correlação significativa e positiva $(p<0.05)$ da Frequência de treino com as dimensões de DE continuidade $(r=0,16)$, redução de outras atividades $(r=0,27)$, falta de controle $(r=0,24)$, tempo $(\mathrm{r}=0,25)$ e escore geral de $\mathrm{DE}(\mathrm{r}=0,25)$. A análise de regressão múltipla revelou que apenas a frequência de treino apresentou associação significativa e positiva com as dimensões de DE. Especificamente, a frequência semanal de treino explicou $1 \%$ da variância da continuidade, $6 \%$ da redução de outras atividades, $5 \%$ da falta de controle, $5 \%$ do tempo e $7 \%$ do escore geral de DE. Concluiu-se que a frequência semanal de treino parece ser um fator interveniente no grau de DE em atletas recreacionais.

Palavras chave: Comportamento Dependente; Dependência ao Exercício; Psicologia do Exercício; Exercício

\section{RESUMEN}

Este estudio transversal tuvo como objetivo investigar el papel predictivo de la edad, la frecuencia de entrenamiento y el tiempo de práctica en el grado de dependencia del ejercicio (DE) de los atletas recreativos. Participaron 159 atletas recreativos de atletismo, musculación y crossfit, con una edad media de $31.59 \pm 7.98$ años, del estado de Pernambuco. Los instrumentos utilizados fueron un cuestionario socio demográfico y la Exercise Dependence ScaleRevised. El análisis de los datos se realizó utilizando la correlación de Spearman y la Regresión Múltiple (p <0.05). 


\section{Freire et al.}

Los resultados mostraron una correlación significativa y positiva $(\mathrm{p}<0.05)$ de la Frecuencia de entrenamiento con continuidad $(\mathrm{r}=0.16)$, reducción en otras actividades $(\mathrm{r}=0.27)$, falta de control $(\mathrm{r}=0.24)$, tiempo $(\mathrm{r}=0.25)$ y puntaje general de $\mathrm{DE}(\mathrm{r}=0.25)$. El análisis de regresión múltiple reveló que solo la frecuencia de entrenamiento tenía una predicción significativa y directa sobre las dimensiones de la DE. Específicamente, la frecuencia de entrenamiento semanal explica el 1\% de la variación de continuidad, el 6\% de la reducción en otras actividades, el 5\% de la falta de control, el 5\% del tiempo y el 57\% de la puntuación general de DE. se concluye que la frecuencia semanal de entrenamiento parece ser un factor interviniente en el grado de DE en los atletas recreativos.

Palabras clave: Comportamiento Dependiente; Dependencia al Ejercicio; Psicología del Ejercicio; Ejercicio.

\section{ABSTRACT}

This cross-sectional study aimed to investigate the association of age, frequency of training and time of practice with the degree of dependence on exercise (DE) of recreational athletes. 159 recreational running, bodybuilding and crossfit athletes participated, with an average age of $31.59 \pm 7.98$ years, from the state of Pernambuco. The instruments used were a sociodemographic questionnaire to characterize the sample and the Exercise Dependence Scale-Revised to measure ED. Data analysis was conducted using Spearman's Correlation and Multiple Regression $(\mathrm{p}<0.05)$. The results showed a significant and positive correlation $(\mathrm{p}<0.05)$ of the training frequency with the dimensions of DE continuity $(r=0.16)$, reduction in other activities $(r=0.27)$, lack of control $(r=0,24)$, time $(r=$ $0.25)$ and general DE score $(r=0.25)$. The multiple regression analysis revealed that only the training frequency had a significant and positive prediction on the dimensions of ED. Specifically, the weekly training frequency explains $1 \%$ of the continuity variance, $6 \%$ of the reduction in other activities, $5 \%$ of the lack of control, $5 \%$ of the time and $57 \%$ of the general ED score. It is concluded that the weekly frequency of training seems to be an intervening factor in the degree of ED in recreational athletes.

Keywords: Dependent Behaviour; Exercise Addiction; Exercise Psychology; Exercise.

\section{INTRODUCCIÓN}

A prática excessiva do exercício físico pode levar ao desenvolvimento de comportamentos patológicos e dependentes, como a dependência de exercício (DE) (Nogueira, Molinero, Salguero, \& Márquez, 2018). A DE que se caracteriza como uma ânsia pelo exercício físico e um comportamento incontrolável em praticálo de forma excessiva, que se manifesta por sintomas fisiológicos de intolerância e abstinência e/ou psicológicos, como ansiedade e depressão (Di Lodovico, Poulnais, \& Gorwood, 2019). Entretanto, ainda são escassos os estudos a respeito da prevalência da DE na população, suas causas e consequentemente suas consequências, fato que pode justificar a ausência deste comportamento dependente na quinta edição do Diagnostic and Statistical Manual of Mental Disorders (Association, 2013), ficando apenas identificada como "comportamento repetitivo" (Hausenblas \& Downs, 2002; Hausenblas \& Giacobbi Jr, 2004).

Nesse cenário, os sujeitos dependentes tratam a prática de exercício como prioridade, abdicando de diversos aspectos (e.g. família, saúde, amigos) para se exercitar
(Almeida, Borba, \& Santos, 2018; Rudolph, 2018; Zeulner, Ziemainz, Beyer, Hammon, \& Janka, 2016). A DE pode ser classificada como primária, quando os indivíduos incluem o uso do exercício como estratégia de coping para as preocupações com a saúde, aparência e estressores em geral (Di Lodovico et al., 2019), ou dependência secundária, quando está incluída na etiologia dos transtornos alimentares (Bóna, Szél, Kiss, \& Gyarmathy, 2019), distorção da autoimagem (Neves, 2015) e da dismorfia corporal (Orrit, 2019; Pope, Phillips, \& Olivardia, 2000; Pope Jr, Gruber, Choi, Olivardia, \& Phillips, 1997), nos quais o exercício físico é elemento compensatório ou mantenedor do transtorno.

Nessa perspectiva, a DE pode trazer danos para a saúde física e mental tanto para atletas quanto para não-atletas (Costa, Della Torre, \& Alvarenga, 2015; Manore, Meyer, \& Thompson, 2018), visto que a excessiva carga de exercício requer uma alta demanda técnica, física e psicológica para os praticantes (Moreira, Mazzardo, Vagetti, Oliveira, \& Campos, 2017), os quais realizam grande volume de treinamento esperando alcançar os resultados 


\section{Semanal de treino e dependência ao exercício em atletas recreacionais}

esperados, sejam esses relacionados ao desempenho ou a estética.

Embora a literatura aponte a relação positiva entre a DE e o aumento da prática de exercício (Corazza et al., 2019; Di Lodovico et al., 2019; Lukács, Sasvári, Varga, \& Mayer, 2019), bem como a relação desse comportamento como a idade, praticantes mais jovens estão mais suscetíveis a desenvolver níveis elevados de DE(Di Lodovico et al., 2019; Lukács et al., 2019; Nogueira et al., 2018; Zeulner et al., 2016), ainda não existe um consenso a respeito do papel do volume e frequência de treino sobre tal comportamento dependente em atletas recreacionais e praticantes de exercício(Di Lodovico et al., 2019; Lukács et al., 2019; Nogueira et al., 2018).

Considerando o crescimento exponencial de praticantes recreacionais de exercício físico nos últimos anos, principalmente em modalidades como a musculação (bodybuiling), o crossfit e a corrida (Esteve-Lanao et al., 2017), e que a DE é um fenômeno grave que requer atenção, é de suma importância identificar possíveis fatores que podem estar relacionados a esse comportamento dependente para que treinadores, psicólogos e praticantes estejam atentos ao comportamento do praticante/atleta em relação ao exercício.

Desta forma, o presente estudo teve como objetivo foi investigar a associação da idade, frequência de treino e tempo de prática sobre o grau de DE de atletas recreacionais. A hipótese inicial é que a frequência semanal de treino e o tempo de prática apresentarão maior associação com o $\mathrm{DE}$ dos praticantes de exercício do que a idade.

\section{MATERIAL Y MÉTODOS}

\section{Desenho do estudo}

Trata-se de um estudo descritivo com delineamento transversal e investigação metodológica (Ato, Lopez, \& Benavente, 2013). O estudo foi desenvolvido por meio das diretrizes do Strengthening the Reporting of Observational Studies in Epidemiology (STROBE) (Malta, Cardoso, Bastos, Magnanini, \& Silva, 2010) para estudos observacionais.

\section{Participantes}

Participaram da pesquisa 159 atletas recreacionais de corrida de rua $(n=38)$, crossfit $(n=85)$ e musculação $(\mathrm{n}=35)$, de ambos os sexos (77 homens e 81 mulheres). Os participantes tinham média de idade de $31,59(\mathrm{dp}=7,98)$ anos, tempo médio de prática de 1,68 $(\mathrm{dp}=0,87)$ anos e frequência semanal de treino de 4,62 dias ( $\mathrm{dp}=1,15)$. Os critérios de inclusão foram: 1$)$ > 18 anos; 2) ser praticante de exercício físico há pelo menos três meses; e 3) treinar regularmente pelo menos duas vezes por semana. Apenas os indivíduos que assinaram o termo de consentimento livre e esclarecido participaram deste estudo.

\section{Instrumentos}

Variáveis independentes

As variáveis independentes do estudo foram mensuradas a partir de um questionário semiestruturado elaborada pelos próprios autores com questões referentes à modalidade, idade, tempo de prática e frequência semanal de treinos.

Variável dependente

A variável dependente do estudo foi a $\mathrm{DE}$, que foi mensurada por meio da Exercise Dependence ScaleRevised (EDS-R), que foi desenvolvida por Downs, Hausenblas e Nigg (2004) e validada para o contexto brasileiro por Alchieri et al. (2015). A EDS-R avalia a dependência ao exercício por meio de 21 itens, atribuindo-se a cada item um escore de um a seis, representando nunca e sempre, respectivamente, conforme comportamentos ou crenças nos últimos três meses. O somatório desses itens possibilita o cálculo de sete escores diferenciados, cada um representando um sintoma da dependência de exercício, a saber: a abstinência (Refere-se à prática de exercício físico superior à sua intenção de fazê-lo), a continuidade (Este fator representa uma continuidade dos exercícios, mesmo quando são contraindicados), a tolerância (Este termo, utilizado no campo fisiológico, refere-se à necessidade de aumentar a carga o exercício para sentir-se satisfeito), a perda de controle (Este fator indica uma falta de controle do indivíduo na prática de exerício, tendo que manter sempre a intensidade, duração e frequência do exercício para se sentir satisfeito), a redução de outras atividades (Esta dimensão diz respeito à redução do convívio com familiares e amigos para passar tempo praticando exercício), o tempo (Refere-se ao tempo despendido 


\section{Freire et al.}

pelo indivíduo na prática de exercício) e os efeitos intencionais (Propõe que, quando privado de se exercitar, o indivíduo passa a sentir sintomas característicos de abstinência, como ansiedade, irritabilidade e tensão). Além disso, o instrumento também possibilita o cálculo de um escore global representativo da dependência ao exercício por meio da média dos valores de todos os itens. Quanto maior o escore total, mais alto é o nível de dependência ao exercício e quanto maior o escore em determinado sintoma, mais comprometido o indivíduo se encontra naquele aspecto da dependência de exercício. O escore total da EDS-R é interpretado enquanto um continuum (pontuação mínima 21 e máxima 105) no qual escores mais elevados indicam maiores sintomas de DE, acima de 70 pontos foi considerado elevado e abaixo de 70 pontos foi considerado baixo (Alchieri et al., 2015). O Alfa de Cronbach variou entre 0,76 e 0,87, indicando forte consistência dos dados (Hair, Risher, Sarstedt e Ringle, 2019).

\section{Procedimiento}

Os procedimentos adotados nesta pesquisa estão de acordo com os critérios de ética em pesquisa com seres humanos de acordo com a resolução (466/12) do Conselho Nacional de Saúde. Inicialmente, o contato era com a gerência dos centros de treinamentos (academias, box de corssfit, acessórias de grupo de corrida) para obter autorização para coleta de dados. Em seguida, o Comitê de Ética em Pesquisa da Universidade Federal do Vale do São Francisco aprovou o estudo (protocolo 2.442.590). Os participantes foram selecionados de forma nãoprobabilística e por conveniência e a coleta de dados se deu por meio de formulário online disponibilizado pelo Survey Monkay. Os sujeitos que tiveram o interesse em participar da pesquisa fizeram o aceite por meio do Termo de Consentimento Livre e Esclarecido no formulário online, assinalando "concordo". O endereço eletrônico foi desenvolvido para alojar o questionário eletrônico destinado ao estudo e foi divulgado via e-mail e redes sociais (Facebook, Instagram e Whats App). A plataforma para preenchimento dos questionários ficou disponível para a recepção das respostas dos sujeitos por 90 dias. Antes do preenchimento, uma breve instrução era dada aos participantes contendo informações referentes aos objetivos da pesquisa, o público-alvo e a estimativa de tempo para finalizar o questionário (aproximadamente 15 minutos).

\section{Análise dos Dados}

A análise de dados foi realizada através de estatísticas descritivas e inferenciais. A análise preliminar dos dados foi realizada por meio do teste de normalidade de Kolmogorov-Smirnov e o teste de homogeneidade das variâncias de Levene. Como os dados apresentaram distribuição normal, a média e o desvio padrão foram usados para a caracterização dos resultados. A correlação de Pearson foi usada para investigar a associação da idade, tempo de prática e frequência semanal de treino com as dimensões de DE em praticantes de exercício. O modelo de regressão múltipla foi usado para determinar se idade, tempo de prática e frequência semanal (variáveis independentes) estão associadas às dimensões de DE (variáveis dependentes). O modelo foi conduzido usando o método Backward para inserir as variáveis (critério de remoção $F=0,10$ ). Todas as variáveis independentes foram inseridas juntas nos modelos. Não houve correlações suficientemente fortes entre variáveis que indicassem problemas com multicolinearidade (VIF $<5,0)$. Todas as análises foram realizadas no SPSS v. 22. 0.

\section{RESULTADOS}

A maior parte da amostra $(51,3 \%)$ foi composta pelo sexo feminino e de participantes $(51,3 \%)$ está entre a faixa etária de 18 a 30 anos. De acordo com a modalidade, a maioria dos respondentes pratica crossfit $(53,8 \%)$. Ainda, 44,3\% participantes praticava a modalidade há menos de 2 anos e em relação a frequência de treino, percebe-se que a maioria dos participantes treina 5-7 vezes por semana $(56,3 \%)$.

Os resultados apresentados na Tabela 1 mostram que os valores relatados sobre a DE variaram entre 1,00 e 5 , e os valores da assimetria e kurtosis variaram entre 0,06 e 0,13 , respectivamente. Os valores médios das variáveis variaram entre 2,20 e 3,82 .

Tabela 1. Valores descritivos (mínimo, máximo, média, desvio-padrão, assimetria e kurtosis) e distribuição das variáveis.

Variáveis $\quad$ Mín. Máx. M (DP) Alfa




\section{Semanal de treino e dependência ao exercício em atletas recreacionais}

\begin{tabular}{lllll} 
Intensionalidade & 1,00 & 5,00 & $2,51(1,12)$ & 0,76 \\
Continuidade & 1,00 & 5,00 & $2,20(1,04)$ & 0,79 \\
Tolerância & 1,00 & 5,00 & $3,18(0,96)$ & 0,80 \\
Redução de & 1,00 & 5,00 & $2,38(0,86)$ & 0,76 \\
Outras Atividades & & & & \\
Falta de Controle & 1,00 & 5,00 & $3,82(0,76)$ & 0,80 \\
Abstinência & 1,00 & 5,00 & $3,37(0,87)$ & 0,87 \\
Tempo & 1,00 & 5,00 & $2,51(1,02)$ & 0,81 \\
Escore Geral DE & 1,00 & 5,00 & $2,85(0,62)$ & 0,79 \\
\hline
\end{tabular}

continuidade $(\mathrm{r}=0,16)$, redução de outras atividades $(\mathrm{r}=0,27)$, falta de controle $(\mathrm{r}=0,24)$, tempo $(\mathrm{r}=0,25)$ e escore geral de DE $(r=0,25)$.

Foi realizada uma regressão múltipla com todos os possíveis fatores associados (idade, tempo de prática e frequência de treino) para cada uma das dimensões de DE. A tabela 3 apresenta os modelos finais apenas com os fatores associados que foram retidos no modelo final da regressão e, portanto, mostraram resultados significativos $(\mathrm{p}<0,05)$.

Os resultados obtidos (Tabela 3) indicam que a 'frequência de treino' apresentou associação

Tabela 2. Correlação entre as variáveis sociodemográficas com dependência do exercício.

\begin{tabular}{|c|c|c|c|c|c|c|c|c|c|c|c|}
\hline Variáveis & 1 & 2 & 3 & 4 & 5 & 6 & 7 & 8 & 9 & 10 & 11 \\
\hline 1.Idade & & $0,40 * *$ & $-0,23 * *$ & 0,07 & $-0,02$ & $-0,05$ & 0,04 & $-0,08$ & $-0,10$ & $-0,01$ & $-0,02$ \\
\hline 2.Tempo de Prática & & & 0,11 & $-0,01$ & 0,09 & 0,03 & 0,10 & 0,10 & $-0,00$ & 0,07 & 0,08 \\
\hline 3.Frequência de Treino & & & & 0,10 & $\mathbf{0 , 1 6 *}$ & 0,13 & $0,27 * *$ & $0,24 * *$ & 0,01 & $0,25 * *$ & $0,25 * *$ \\
\hline 4.Intensionalidade & & & & & $0,29 * *$ & $0,38 * *$ & $0,52 * *$ & $0,20 * *$ & $0,28 * *$ & $0,60 * *$ & $0,74 * *$ \\
\hline 5.Continuidade & & & & & & $0,40 * *$ & $0,40 * *$ & 0,11 & $0,22 * *$ & $0,37 * *$ & $0,63 * *$ \\
\hline 6.Tolerância & & & & & & & $0,32 * *$ & $0,38 * *$ & $0,24 * *$ & $0,36 * *$ & $0,67 * *$ \\
\hline 7. Redução de Outras & & & & & & & & $0,27 * *$ & $0,23 * *$ & $0,66^{* *}$ & $0,74 * *$ \\
\hline \multicolumn{12}{|l|}{ Atividades } \\
\hline 8. Falta de Controle & & & & & & & & & $0,25 * *$ & $0,16^{*}$ & $0,48 * *$ \\
\hline 9. Abstinência & & & & & & & & & & $0,19 *$ & $0,51 * *$ \\
\hline 10.Tempo & & & & & & & & & & & $0,75^{* *}$ \\
\hline 11.Escore Geral DE & & & & & & & & & & & \\
\hline
\end{tabular}

Correlação de Pearson ** A correlação é significativa a nível $0,01 . *$ A correlação é significativa a nível 0,05 . significativa $(\mathrm{p}<0,05)$ e positiva com a maioria das dimensões de DE. A combinação destas variáveis explicou $1 \%$ da continuidade $(\beta=0,16), 6 \%$ da redução de outras atividades $(\beta=0,27), 5 \%$ da falta de controle $(\beta=0,24), 5 \%$ do tempo $(\beta=0,25)$ e $7 \%$ do escore geral de $\mathrm{DE}(\beta=0,25)$.

A análise das correlações apresentadas na Tabela 2 indicou as seguintes correlações significativas $(\mathrm{p}<0,01)$ e $(\mathrm{p}<0,05)$. Frequência de treino com

Tabela 3. Modelos finais das regressões lineares avaliando a capacidade preditiva das variáveis sociodemográficas (idade, tempo de prática e frequência de treino) sobre as dimensões de DE

\begin{tabular}{lcccccccc}
\hline Dimensão Predita & $\mathrm{R}$ & $\mathrm{R}^{2}$ & $\mathrm{~F}$ & Sig. & Fatores preditores & $\beta$ & $\mathrm{VIF}$ & $\mathrm{DW}$ \\
\hline Intencionalidade & - & - & - & - & - & - & - & - \\
Continuidade & 0,16 & 0,01 & 4,099 & 0,045 & Frequência de Treino & 0,16 & 0,98 & 1.796 \\
Tolerância & - & - & - & - & - & - & - & -
\end{tabular}




\section{Freire et al.}

\begin{tabular}{lcccccccc}
$\begin{array}{l}\text { Redução de outras } \\
\text { atividades }\end{array}$ & 0,27 & 0,06 & 12,384 & 0,001 & Frequência de Treino & 0,27 & 0,98 & 1.801 \\
Falta de controle & 0,24 & 0,05 & 9,991 & 0,002 & Frequência de Treino & 0,24 & 0,98 & 1.865 \\
Abstinência & - & - & - & - & - & - & - & - \\
Tempo & 0,25 & 0,05 & 10,695 & 0,001 & Frequência de Treino & 0,25 & 0,98 & 1.900 \\
Escore Geral DE & 0,25 & 0,07 & 10,556 & 0,001 & Frequência de Treino & 0,25 & 0,98 & 1.900 \\
\hline
\end{tabular}

Nota: Em todos os casos, as variáveis iniciais nos modelos foram: 'idade', 'tempo de prática' e 'frequência de treino'. Na tabela são apresentadas apenas as variáveis retidas nos modelos finais, utilizando o método Backward. DW= Durbin-Watson.

\section{DISCUSSÃO}

A presente investigação visou avaliar a idade, o tempo de prática e a frequência de treino como fatores associados da DE em praticantes de exercício. Os principais resultados revelaram que apenas a frequência de treino apresentou associação com a DE (continuidade, redução de outras atividades, falta de controle, tempo e escore geral de DE) (Tabela 3).

O principal resultado desta investigação responde uma das hipóteses iniciais demostrando que a frequência de treino está associada positivamente com o desenvolvimento da DE em praticantes de exercício (Tabela 3). Ressalta-se que a frequência semanal de treino apresentou associação positiva com diversas dimensões da $\mathrm{DE}$, indicando que quanto mais vezes na semana o indivíduo treina, maior pode ser a predisposição de praticar exercícios além do planejado e com grande quantidade de tempo, de reduzir o convívio social, ocupacional ou de lazer para se exercitar, além de apresentarem maior incapacidade de reduzir sua carga de exercícios, mesmo quando são contraindicados (Alchieri et al., 2015; Clifford \& Blyth, 2019; Nogueira et al., 2018). Este achado parece indicar que o aumento do volume de treinamento do atleta amador/recreacional pode levar a perda do controle sobre os limites físicos e mentais $\mathrm{e}$, consequentemente, desencadeando a DE (Di Lodovico et al., 2019; Nogueira et al., 2018). O fato de muitos atletas amadores não possuírem acompanhamento diário de treinador e equipe multidisciplinar pode estar relacionado a tal resultado, uma vez que o treinador tem papel fundamental no controle da carga de treinamento diária e semanal (Impellizzeri et al., 2006).

Estudos recentes (De La Vega, Parastatidou, RuizBarquin, \& Szabo, 2016; Lucidi et al., 2016; SchiphofGodart \& Hettinga, 2017) demostraram relação positiva entre o risco de desenvolvimento da DE e a intensidade e a frequência da prática do, verificando que quanto maior a frequência de treino maior os indicativos de DE. Clifford e Blyth (2019) demostraram que atletas/estudantes com maior frequência semanal de treino revelaram maior predisposição para a prática de exercícios além do planejado pelos treinadores. Lukács et al. (2019) observaram que corredores amadores tendem a aumentar o volume e a intensidade das suas atividades para alcançarem maiores níveis de satisfação/prazer. Desta forma, os achados da presente investigação revelam que a frequência de treino pode ser considerada um fator prejudicial para a saúde física e mental, uma vez que pode levar ao desencadeamento da DE e, consequentemente, a adoção de outros comportamentos dependentes e patológicos (e.g. Transtorno alimentar, distorção da imagem corporal, uso de esteroides anabolizantes) (Di Lodovico et al., 2019).

A literatura aponta que existem mecanismos psicológicos que podem favorecer o aumento da prática do exercício (e.g. paixão pela prática, estratégias de coping) (Di Lodovico et al., 2019; Lukács et al., 2019; Nogueira et al., 2018) e, consequentemente, desencadear a DE. Pesquisas recentes afirmam que a paixão pelo exercício é um atributo psicológico que pode levar ao aumento do volume da prática do exercício e, consequentemente, ao desencadeamento da DE (Back, Josefsson, Ivarsson, \& Gustafsson, 2019; Kovacsik, Soós, de la Vega, Ruíz-Barquín, \& Szabo, 2018), uma vez que quando o indivíduo pratica a atividade pela própria vontade, por prazer e apresenta identificação com a prática, existe uma tendência de maior investimento de tempo e energia por parte do praticante (Back et al., 2019; Kovacsik et al., 2018). No entanto, o presente estudo não abordou a associação da $\mathrm{DE}$ com este 


\section{Semanal de treino e dependência ao exercício em atletas recreacionais}

atributo psicológico, o que é uma limitação desta pesquisa.

Embora a frequência semanal de treino tenha se mostrado como um fator preditor da DE, o tempo de pratica não apresentou predição significativa com as dimensões e o escore geral de DE (Tabela 3), indicando que o tempo de prática/experiência por si só parece não levar ao desenvolvimento de DE. Esses achados não corroboram com a literatura (Downs, Savage, \& DiNallo, 2013; Warburton \& Bredin, 2017), uma vez que diversos estudos apontam que o tempo de prática pode ser um fator intermitente para o desenvolvimento da DE (Downs et al., 2013; Warburton \& Bredin, 2017). Downs et al. (2013) investigaram que jovens fisicamente ativos apresentam sinais de patológicos de exercício físico, que podem levar ao desenvolvimento de DE. Warburton e Bredin (2017) observaram que o comportamento esportivo ativo na infância pode favorecer a um engajamento de comportamentos dependentes (eg. DE) na fase adulta. Esse fato pode estar relacionado ao fato de alguns praticantes de exercício utilizarem a prática de exercício para superar outro vício (e.g. Depressão, alcoolismo, tabagismo) ou como maneira de reduzir o estresse, sendo a forte dedicação transformando os praticantes em viciados pela prática do exercício (Lee et al., 2017; Warburton \& Bredin, 2017).

Percebeu-se que a idade também não se mostrou como um fator preditor para o desenvolvimento da DE (Tabela 3), evidenciando que a idade cronológica não parece interferir no comportamento do indivíduo em relação à prática do exercício. Esses achados podem ser considerados contraditórios, na medida em que pesquisas afirmam que a idade pode ser um fator para o desencadeamento de comportamentos dependentes (e.g. DE; uso de anabolizantes transtorno alimentar) (Clifford \& Blyth, 2019; Lichtenstein \& Jensen, 2016). Lichtenstein e Jensen (2016) observaram que os praticantes de crossfit do sexo masculino e jovens apresentaram maior DE que seus respectivos pares. No entanto, vale ressaltar que fatores culturais (e.g modalidade, hábitos, costumes) também podem interferir diretamente nesses comportamentos (Fidan, Ertekin, Işikay, \& Kırpınar, 2010).

Apesar das contribuições relevantes obtidas a partir dos resultados deste estudo, algumas limitações precisam ser destacadas. Primeiramente, por se tratar de um estudo transversal e com único instrumento (escala de auto relato), os dados obtidos permitem correlações entre variáveis, mas não inferências de causalidade, o que é uma das limitações da pesquisa. Vale destacar também o reduzido número de participantes e que eram praticantes de apenas três modalidades de exercício, o que impossibilita a generalização dos resultados para praticantes de outras modalidades, embora traga implicações relevantes para os profissionais envolvidos com a prescrição de exercício. Assim, futuras pesquisas devem ampliar o estudo com praticantes de outras culturas, hábitos e de outras modalidades individuais e coletivas, assim como analisar variáveis psicológicas relacionadas à DE que não foram analisadas nesse estudo (e.g. paixão, ansiedade). Por fim, vale destacar que a presente investigação não avaliou se os atletas tinham treinador/acompanhante durante os treinamentos.

\section{CONCLSÃO}

Pode-se concluir que a frequência semanal de treino está associada positivamente, mas com intensidade baixa, com o grau de DE em atletas recreacionais. Diante disso, a orientação e acompanhamento de treinadores/profissionais de educação física torna-se importante com o intuito de controlar o volume e intensidade da carga de treinamento, uma vez que o aumento do volume e intensidade de treino semanal sem orientação profissional pode levar ao desenvolvimento da DE em atletas, ocorrência de lesões e outros prejuízos físicos e mentais.

\section{REFERENCIAS (APA $7^{\text {a }}$ EDICION)}

Alchieri, J. C., Gouveia, V. V., Oliveira, I. C. V. d., Medeiros, E. D. d., Grangeiro, A. S. d. M., \& Silva, C. (2015). Exercise Dependence Scale: adaptação e evidências de validade e precisão. J Bras Psiquiatr, 64(4), 279-287.

\section{https://doi.org/10.1590/0047-2085000000090}

Almeida, C., Borba, V. V., \& Santos, L. (2018). Orthorexia nervosa in a sample of Portuguese fitness participants. Eating and Weight Disorders-Studies on Anorexia, Bulimia and Obesity, 23(4), 443-451.

https://doi.org/10.1007/s40519-018-0517-y

Association, A. P. (2013). Diagnostic and Statistical Manual of Mental Disorders, 5th edn (Washington, DC: APA). 


\section{Freire et al.}

Ato, M., Lopez, J. J., \& Benavente, A. (2013). A classification system for research designs in psychology. anales de psicología, 29(3), 1038-1059.

Back, J., Josefsson, T., Ivarsson, A., \& Gustafsson, H. (2019). Psychological risk factors for exercise dependence. International Journal of Sport and Exercise Psychology, 1-12.

https://doi.org/10.1080/1612197X.2019.1674902

Bóna, E., Szél, Z., Kiss, D., \& Gyarmathy, V. A. (2019). An unhealthy health behavior: analysis of orthorexic tendencies among Hungarian gym attendees. Eating and Weight Disorders - Studies on Anorexia, Bulimia and Obesity, 24(1), 13-20.

https://doi.org/10.1007/s40519-018-0592-0

Clifford, T., \& Blyth, C. (2019). A pilot study comparing the prevalence of orthorexia nervosa in regular students and those in University sports teams. Eating and Weight Disorders-Studies on Anorexia, Bulimia and Obesity, 24(3), 473-480.

https://doi.org/10.1007/s40519-018-0584-0

Corazza, O., Simonato, P., Demetrovics, Z., Mooney, R., van de Ven, K., Roman-Urrestarazu, A., . . . Santacroce, R. (2019). The emergence of Exercise Addiction, Body Dysmorphic Disorder, and other image-related psychopathological correlates in fitness settings: A cross sectional study. PloS one, 14(4), e0213060.

https://doi.org/10.1371/journal.pone.0213060

Costa, A. C. P., Della Torre, M. C. d. M., \& dos Santos Alvarenga, M. (2015). Atitudes em relação ao exercício e insatisfação com a imagem corporal de frequentadores de academia. Revista Brasileira de Educação Física e Esporte, 29(3), 453-464.

https://doi.org/10.1590/1807-55092015000300453

De La Vega, R., Parastatidou, I. S., Ruiz-Barquin, R., \& Szabo, A. (2016). Exercise addiction in athletes and leisure exercisers: the moderating role of passion. Journal of behavioral addictions, 5(2), 325-331.

https://doi.org/10.1556/2006.5.2016.043

Di Lodovico, L., Poulnais, S., \& Gorwood, P. (2019). Which sports are more at risk of physical exercise addiction: A systematic review. Addictive behaviors, 93, 257-262. https://doi.org/10.1016/j.addbeh.2018.12.030

Downs, D. S., Hausenblas, H. A., \& Nigg, C. R. (2004). Factorial validity and psychometric examination of the Exercise Dependence ScaleRevised. Measurement in Physical Education and Exercise Science, 8(4), 183-201.

https://doi.org/10.1207/s15327841mpee0804_1

Downs, D. S., Savage, J. S., \& DiNallo, J. M. (2013). Self-determined to exercise? Leisure-time exercise behavior, exercise motivation, and exercise dependence in youth. Journal of Physical Activity and Health, 10(2), 176-184.

https://doi.org/10.1123/jpah.10.2.176

Esteve-Lanao, J., Moreno-Pérez, D., Cardona, C. A., Larumbe-Zabala, E., Muñoz, I., Sellés, S., \& Cejuela, R. (2017). Is Marathon training harder than the ironman training? an ECO-method comparison. Front Physiol, 8, 298.

https://doi.org/10.3389/fphys.2017.00298

Fidan, T., Ertekin, V., Işikay, S., \& Kırpınar, I. (2010). Prevalence of orthorexia among medical students in Erzurum, Turkey. Comprehensive psychiatry, 51(1), 49-54.

https://doi.org/10.1016/j.comppsych.2009.03.001

Hair, J. F., Risher, J. J., Sarstedt, M., \& Ringle, C. M. (2019). When to use and how to report the results of PLS-SEM. European Business Review, 31(1), 2-24.

https://doi.org/10.1108/EBR-11-2018-0203

Hausenblas, H. A., \& Downs, D. S. (2002). How much is too much? The development and validation of the exercise dependence scale. Psychology and health, 17(4), 387-404.

https://doi.org/10.1080/0887044022000004894

Hausenblas, H. A., \& Giacobbi Jr, P. R. (2004). Relationship between exercise dependence symptoms and personality. Personality and Individual Differences, 36(6), 1265-1273.

https://doi.org/10.1016/S0191-8869(03)00214-9

Impellizzeri, F. M., Marcora, S. M., Castagna, C., Reilly, T., Sassi, A., Iaia, F., \& Rampinini, E. (2006). Physiological and performance effects of generic versus specific aerobic training in soccer players. Int J Sports Med, 27(06), 483-492. 


\section{Semanal de treino e dependência ao exercício em atletas recreacionais}

https://doi.org/10.1055/s-2005-865839

Kovacsik, R., Soós, I., de la Vega, R., Ruíz-Barquín, R., \& Szabo, A. (2018). Passion and exercise addiction: Healthier profiles in team than in individual sports. International Journal of Sport and Exercise Psychology, 1-11.

https://doi.org/10.1080/1612197X.2018.1486873

Lee, D.-c., Brellenthin, A. G., Thompson, P. D., Sui, X., Lee, I.-M., \& Lavie, C. J. (2017). Running as a key lifestyle medicine for longevity. Progress in cardiovascular diseases, 60(1), 45-55.

https://doi.org/10.1016/j.pcad.2017.03.005

Lichtenstein, M. B., \& Jensen, T. T. (2016). Exercise addiction in CrossFit: Prevalence and psychometric properties of the Exercise Addiction Inventory. Addictive Behaviors Reports, 3, 33-37.

https://doi.org/10.1016/j.abrep.2016.02.002

Lucidi, F., Pica, G., Mallia, L., Castrucci, E., Manganelli, S., Bélanger, J., \& Pierro, A. (2016). Running away from stress: How regulatory modes prospectively affect athletes' stress through passion. Scandinavian journal of medicine \& science in sports, 26(6), 703-711.

https://doi.org/10.1111/sms.12496

Lukács, A., Sasvári, P., Varga, B., \& Mayer, K. (2019). Exercise addiction and its related factors in amateur runners. Journal of behavioral addictions, 17.

https://doi.org/10.1556/2006.8.2019.28

Malta, M., Cardoso, L. O., Bastos, F. I., Magnanini, M. M. F., \& Silva, C. M. F. P. d. (2010). STROBE initiative: guidelines on reporting observational studies. Revista de saúde pública, 44(3), 559-565.

http://doi.org/10.1590/S0034-89102010000300021

Manore, M. M., Meyer, N. L., \& Thompson, J. L. (2018). Sport nutrition for health and performance: Human Kinetics.

Moreira, N. B., Mazzardo, O., Vagetti, G. C., Oliveira, V. d., \& Campos, W. d. (2017). Associação entre dependência do exercício físico e percepção da qualidade de vida no basquetebol master brasileiro. Revista Brasileira de Ciências do Esporte, 39(4), 433441. https://doi.org/10.1016/j.rbce.2017.07.001

Neves, A. (2015). Body experience as the core of body image development. Body Image: Advances in Psychology Research, 21-36.

Nogueira, A., Molinero, O., Salguero, A., \& Márquez, S. (2018). Exercise Addiction in Practitioners of Endurance Sports: A Literature Review. Frontiers in psychology, 9, 1484.

https://doi.org/10.3389/fpsyg.2018.01484

Orrit, G. (2019). Muscle Dysmorphia: Predictive and protective factors in adolescents. Cuadernos de psicología del deporte, 19(3), 1-11.

https://doi.org/10.6018/cpd.347981

Pope, H., Phillips, K. A., \& Olivardia, R. (2000). O complexo de Adônis: a obsessão masculina pelo corpo: Editora Campus.

Pope Jr, H. G., Gruber, A. J., Choi, P., Olivardia, R., \& Phillips, K. A. (1997). Muscle dysmorphia: An underrecognized form of body dysmorphic disorder. Psychosomatics, 38(6), 548-557.

Rudolph, S. (2018). The connection between exercise addiction and orthorexia nervosa in German fitness sports. Eating and Weight Disorders-Studies on Anorexia, Bulimia and Obesity, 23(5), 581-586.

https://doi.org/10.1007/s40519-017-0437-2

Schiphof-Godart, L., \& Hettinga, F. J. (2017). Passion and pacing in endurance performance. Front Physiol, 8,83 .

https://doi.org/10.3389/fphys.2017.00083

Warburton, D. E., \& Bredin, S. S. (2017). Health benefits of physical activity: a systematic review of current systematic reviews. Current opinion in cardiology, 32(5), 541-556.

https://doi.org/10.1097/HCO.0000000000000437

Zeulner, B., Ziemainz, H., Beyer, C., Hammon, M., \& Janka, R. (2016). Disordered eating and exercise dependence in endurance athletes. Advances in Physical Education, 6(2).

https://doi.org/10.4236/ape.2016.62009 
Freire et al. 


\section{Semanal de treino e dependência ao exercício em atletas recreacionais}

\section{REGLAS PARA LA REDACCIÓN FINAL DE SU MANUSCRITO}

En cuanto a las Referencias y citas, se ruega que realicen una búsqueda bibliográfica en Frontiers in Psychology y Cuadernos de Psicología del Deporte, incluyendo al menos dos referencias de cada una de estas revistas (en su caso), que se ajusten a su trabajo con el fin de potenciar la visibilidad de las revistas nacionales. Además deberán incluir los DOI de todas las referencias bibliográficas que los tengan.

Para la obtención del DOI le rogamos que utilicen la herramienta on-line Simple Text Query disponible en: https://apps.crossref.org/simpleTextQuery

o en su defecto:

https://search.crossref.org/references?utm_term=Link\%20References\&utm_campaign=Simple\%20Text\%20Query \%20for\%20matching\%20references\%20is\%20being\%20replaced\&utm_content=email\&utm_source=Act-

On+Software\&utm_medium=email\&cm_mmc=Act-On\%20Software-_-email-_-

Simple\%20Text\%20Query\%20for\%20matching\%20references\%20is\%20being\%20replaced-_-

Link\%20References

\section{-Lista de Autores.}

IMPORTANTE. Las listas de autores siempre deben ser <Apellido/s>, <Inicial o Nombre> tanto en para autores del artículo como en las referencias para los autores y para editores. Por favor, no usar "\&", "and" o "y" (si, a lo largo del texto)

Ejemplo: Apellido1, N1., Apellido2, N2., Apellido3, N3., Apellido4, N4.

Nota. En caso de que el autor sea una organización se escribirá su nombre completo o sus iniciales (a gusto del autor). Ejemplo: Organización Mundial de la Salud o bien OMS

\section{-Filiación:}

Hace falta que la filiación contenga al menos:

<orgname>: Nombre de la universidad, no de la escuela o facultad. <city>: Ciudad <contry>: Pais

\section{-Citas:}

Cuando la cita tenga 3 autores o más, debe señalarse directamente de la siguiente forma:

Apellido autor 1 et al., (año)

\section{-Referencias Bibliográficas:}

IMPORTANTE: NO INCLUIR REFERENCIAS NO PUBLICADAS. Por ejemplo “En revisión” o “Tesis Doctoral Inédita".

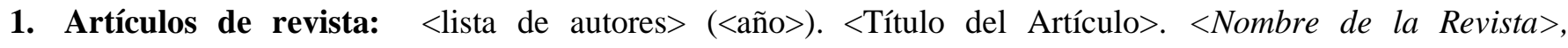
$\langle$ volumen $>(<$ número $>)$, <páginas $>$. <doi >

Si algún dato no se conoce, no se pone. Algunas Posibilidades:

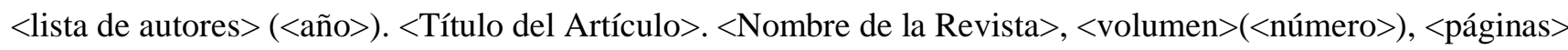

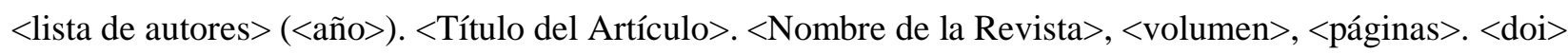




\section{Freire et al.}

$<$ lista de autores $>(<a n ̃ o>) .<$ Título del Artículo>. <Nombre de la Revista $>$, <volumen>, <páginas $>$.

Ejemplo:

Steene-Johannessen, J., Anderssen, S. A., Kolle, E., Andersen, L. B. (2009). Low Muscle Fitness Is Associated with Metabolic Risk in Youth. Medicine \& Science in Sports \& Exercise, 41(7), 1361-1367. https://doi.org/10.1249/MSS.0b013e31819aaae5

2. Libros: <lista de autores $>(<a n ̃ o>)$. <Título del Libro >. <Lugar de Publicación $>$ : $<$ Nombre de la editorial de publicación>

Ejemplo:

Jaenes, J. C., Caracuel, J. C. (2016). Maratón: Preparación Psicológica para el Entrenamiento y la Competición. Sevilla : Almuzara.

3. Capítulos de libro: <lista de autores $>(<$ año $>$ ). $<$ Título del Capítulo $>$ En/In $<$ Lista de editores $>$ (Eds.), $<$ Título del Libro>, <páginas>. <Lugar de Publicación> : <Nombre de la editorial de publicación>

Ejemplo:

Schack, T., Bertollo, M., Koester, D., Maycock, J., Essig, K. (2014). Technological advancements in sport psychology. En Papaioannou, A. G., Hackfort, D. (Eds.), Routledge Companion to Sport and Exercise Psychology, 953-965. London, UK: Routledge

4. Tesis Doctorales: <lista de autores $\rangle(\langle\mathrm{año}\rangle)$. $\langle$ Título de la Tesis $\rangle$. $\langle$ Grado de la tesis, es decir, doctorado, maestría, etc. >, <Escuela o Facultad (opcional)>, 〈Universidad $\rangle,\langle$ Ciudad $\rangle,\langle$ País $\rangle$

Ejemplo:

Carreiro da Costa, F. (1998). O Sucesso pedagógico em educação física: Estudo das condições e factores de ensinoaprendizagem associados ao êxito numa unidade de ensino. Tese de Doutoramento, Instituto Superior de Educação Física, Universidade Técnica de Lisboa, Lisboa, Portugal 